\title{
U-Hopper: User-centric Heteogeneous Opportunistic Middleware
}

\author{
lacopo Carreras and David Tacconi \\ CREATE-NET \\ Via alla Cascata 56/C \\ Trento, Italy \\ name.surname@create-net.org
}

\begin{abstract}
This demonstration presents U-Hopper, a user-centric heterogeneous opportunistic middleware specifically tailored to the diffusion of user centric information, such as contextual and entertainment data, in opportunistic environments. The proposed platform exploits proximity wireless interfaces available on most commercial mobile devices for disseminating data among mobile users. Such diffusion if driven by the specific interests of mobile users, combined with the intrinsic locality of data being generated in such pervasive environments. The prototype is developed over java-enabled smartphones and relies on Bluetooth connectivity for achieving proximity communications. In this paper, U-Hopper is described in all its functional components, together with the details of its software implementation.
\end{abstract}

\section{Keywords}

opportunistic communications, middleware platform, mobile phones, bluetooth, J2ME.

\section{INTRODUCTION}

Opportunistic networking [1] refers to the possibility of delivering data applying an epidemic-like forwarding mechanism, without the need for any dedicated infrastructure. Such communication paradigm received great attention in the last few years as an emerging technology for disseminating data in challenged environments, where due to environmental constraints it is not possible to build an alternative communication infrastructure, or in pervasive environments, where data exchanges are driven by the "social interactions" of mobile users [2]. In particular, the latter case is a direct consequence of the fact that mobile devices (e.g., smartphones or PDAs) are nowadays largely available among people and of the constantly increasing computing, communication and storage power of such devices. Several mobile phones are in fact equipped with Bluetooth, Wi-Fi and Wibree (in the near future), technologies that are directly accessible for programmers through freely available

Permission to make digital or hard copies of all or part of this work for personal or classroom use is granted without fee provided that copies are not made or distributed for profit or commercial advantage and that copies bear this notice and the full citation on the first page. To copy otherwise, to republish, to post on servers or to redistribute to lists, requires prior specific permission and/or a fee.

Bionetics'07 December 10-13, 2007, Budapest, Hungary

Copyright 2007 ICST 978-963-9799-11-0. and easy-to-use APIs. Further, mobile phones are now capable of intensive processing operations and of storing large amounts of data in their internal memory.

However, although opportunistic networking has been deeply investigated from a theoretical point of view, only few real deployments have been proposed, with the main goal of understanding the networking performance of the implemented protocols [3] or the social aspects related to proximity communications [2].

Following these considerations, we have developed a Usercentric Heterogeneous Opportunistic Middleware (U-Hopper), running on any java-enabled smartphone and leveraging Bluetooth connectivity for exchanging data. Such platform combines the user preferences with the requirements imposed by the pervasive services hosted on users' portable device in order to gather and disseminate data. Such process is fully distributed and self-organized, as it does not require any human supervision.

In the proposed demo, we will present a prototype of the U-Hopper platform. The demonstration will comprise three classes of devices. The first one is constituted by sensors embedded in the environment and constantly broadcasting localized information such as snapshots of the conference site or advertisements. Such information is gathered by the second class of devices, which consists of a limited number of smartphones acting as mobile nodes and running the UHopper platform. Through these devices the information will flow around by means of $\mathrm{P} 2 \mathrm{P}$ data exchanges among mobile nodes running the U-Hopper middleware. Finally, information is displayed over a laptop, the third class of devices, which provides a richer user-interface through which the information captured by the U-Hopper platform is displayed.

\section{SYSTEM OVERVIEW}

The considered system architecture consists of two classes of nodes: User-Nodes (U-Nodes), which are resource-rich mobile devices (e.g., smartphones, PDAs) carried around by users during their daily activities, and Tiny-Nodes (TNodes), which are resource-constrained devices embedded in the environment and providing localized information [4]. A part from their technological differences, the 2 classes of devices play a different role in the network. T-Nodes act as providers of information, constantly broadcasting localized information such as advertisements, or snapshots of the surrounding environment. Conversely, U-Nodes act as consumers of information, reading T-Nodes in their communication range, and augmenting pervasive services with such 
data. The data generated by T-Nodes is first stored in the U-Nodes internal memory, and then diffused by means of opportunistic peer-to-peer data exchanges. Users' mobility is therefore exploited in order to achieve system-wide communications. In this demo, we will present a U-Hopper, a

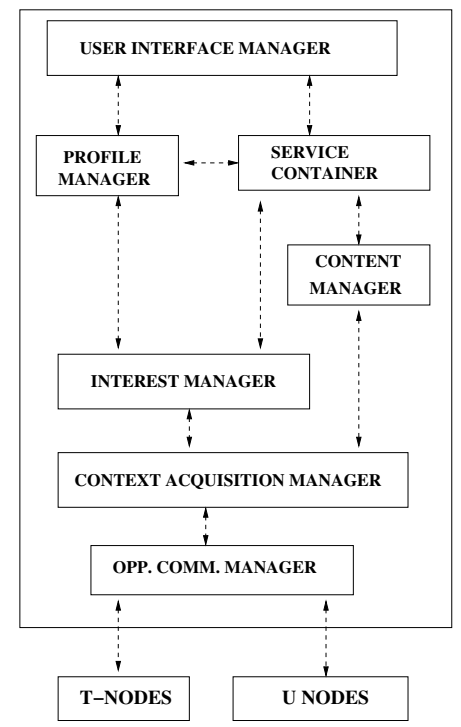

Figure 1: Block diagram and interconnections of the U-Hopper platform.

User-centric Heterogeneous Opportunistic Middleware initially introduced in [5]. Such platform resides on U-Nodes and exploits any proximity communication interface (i.e., Bluetooth, Wi-Fi) in order to (i) gather localized information originating from T-Nodes embedded in the environment (ii) opportunistically disseminate the stored data to other UNodes. The information diffused includes data received from T-Nodes as well as any other information shared by the user (e.g., music, videos, etc.).

As depicted in Fig. 1, the U-Hopper middleware is composed by six distinct components.

The User Interface Manager (UIM) handles any human-tomachine interaction such as data insertion and visualization. The Service Manager (SM) is the execution environment where pervasive services are running. This component allows services deployment, deprecation and update.

The Interest Manager takes into account (i) the user preferences and (ii) the requirements deriving from the pervasive services hosted by the SM, and produces a list of "interests", which are a high-level description of the information the user is interested in. Such interests regulate the way according to which information is exchanged between any 2 U-Nodes accordingly.

The Content Manager (CM) manages the persistent storage available on mobile devices. In particular, it provides context data insertion/deletion, update and search functionalities. In addition, the CM runs appropriate "data aging" algorithms that are needed in order to discard outdated information and preserve the available resources. Such techniques implement information filtering rules that trade off data locality (both in the time and space domains) for available resources (i.e., storage, communication, etc.) [6]. As an example, we can think at a special sale offer ending at $5 \mathrm{pm}$ of the current day. Clearly, as soon as the offer is no longer valid, it is useless to store the corresponding information. The CM is in charge of detecting such situations and of determining when to remove data from the users' device permanent storage.

The Context Acquisition Manager (CAM) takes into account the information deriving from the Interests Manager, and applies data filters on the incoming and outgoing data flows. The Opportunistic Communication Manager (OCM) monitors the availability of data sources in the surrounding environment, and seamlessly performs any networking operation needed for gathering the discovered data. This includes data originating from T-Nodes as well as from other U-Nodes. Please, refer to [5] for a more detailed description of U-Hopper system components.

Fig. 2 depicts the handshake regulating the data exchange between any 2 nodes meeting. The data exchange is triggered by a Node 1 receiving a beacon message used for discovering neighboring peers. In response, Node 1 sends its own interests (Interests $1 \mathrm{MSG}$ ), which are a description of the information Node 1 is interested in. Node 2 responds with the data stored in its own internal memory matching Node 1 interests (DATA 2 MSG), and subsequently, with its own interests (Interests $2 \mathrm{MSG}$ ). Finally, the data exchange is terminated with Node 1 sending any data matching Node 2 interests. The corresponding system components interac-

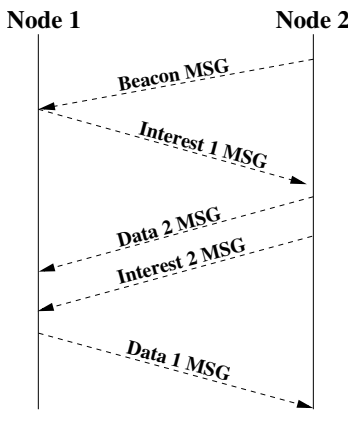

Figure 2: Data handshake between any 2 nodes meeting.

tion flow, when generating the user interests, is presented in Fig. 3. When a beacon message is received by the OCM, a request for the user interests is invoked. Such request is then captured by the IM, which gathers the user profile, the service constraints and returns the user interests. It is worth remarking that the described actions are performed by the U-Hopper platform transparently to the user, thus increasing the system usability of the system, since it does not require any human intervention. On the counterpart, when the interests from an encountered node are received, the data stored in the internal memory is searched accordingly, and information matching the received interests is send back. The corresponding system components interaction flow is depicted in Fig. 4.

\section{IMPLEMENTATION DETAILS}

The main goal of the proposed demo is to show a prototype implementation of the described system over widely diffused software/hardware platforms. In order to embrace the largest number of "potentially available" mobile devices, we selected smartphones as the target platform over which we developed U-Hopper. In fact, smartphones are nowadays 


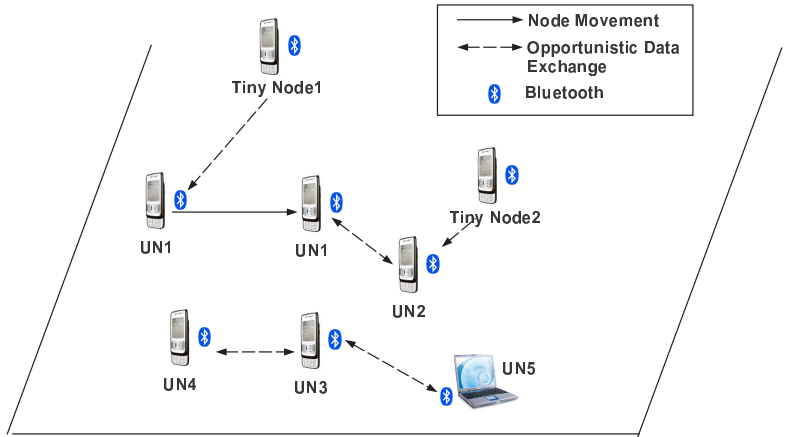

Figure 5: Demonstration scenario.

Context data is then collected by other smartphones (UNodes) passing by, as for instance UN1 gathering data from Tiny Node 1 in Fig. 5. Information is then epidemically diffused by means of opportunistic P2P data exchanges among U-Nodes that take place transparently to the user.

After installing and starting the application, users will be asked to select their preferences. Such preferences will influence the data gathering and data exchange process. Users can then visualize the collected information, such as snapshots and advertisement information, at any time on the smartphone's graphical interface Furthermore, U-Hopper users will have the opportunity to share some information in a totally $\mathrm{P} 2 \mathrm{P}$ fashion, directly acting as source of data. For instance they can share some personal midi files (e.g. ring tones), exchange them with other peers if interested in entertainment information and then listen to the received files through a simple UI. In Fig. 6 two users are shown while using U-Hopper.

In order to show the outcomes of the demo (such as col-

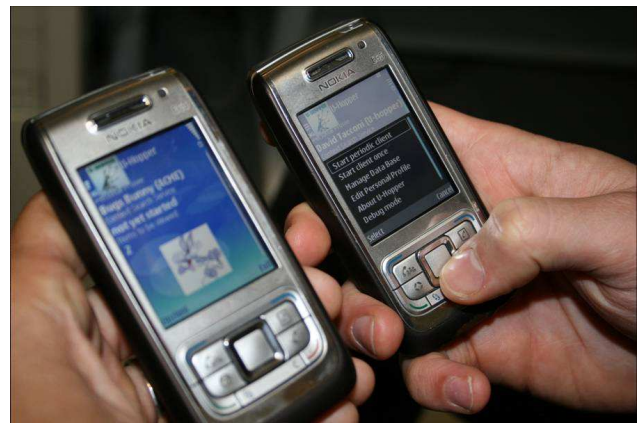

Figure 6: U-Hopper on Nokia E65 smartphones.

lected information and statistics on users P2P communications) in a more user-friendly way, users can visualize at any time such information through an application running on the laptop acting as a user node shown in Fig. 7), available for everyone interested in our application .

\section{CLOSING REMARKS}

The developed U-Hopper is an attempt to provide a reusable software platform capable to transparently handle opportunistic data dissemination among mobile nodes over offthe-shelf hardware and software platforms. Fully distributed pervasive services can leverage such disappearing network in-

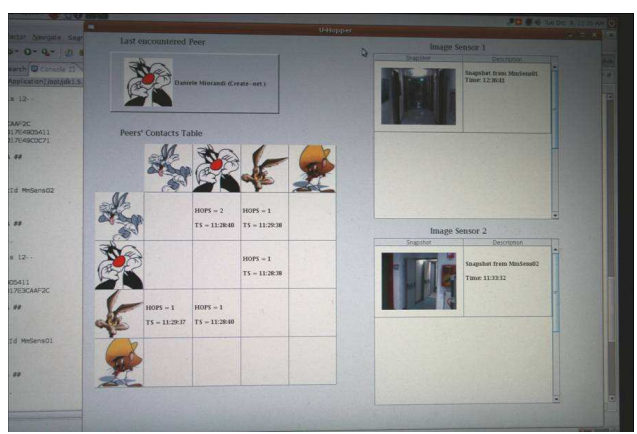

Figure 7: The laptop interface, showing contacts among 4 users and data gathered from T-nodes taking snapshots of the site.

frastructure [4] for retrieving context data without the need of any human supervision. In the presented demo, we have shown a simple application of the U-Hopper middleware. In particular, it is the capability of U-Hopper to exploit the physical mobility of user in order to gather information and diffuse it to other mobile nodes. The platform has been developed over java-enabled smartphones, leveraging Bluetooth connectivity for achieving proximity communications.

\section{ACKNOWLEDGMENTS}

This work has been partially supported by the EC within the framework of the BIONETS project IST-FET-SAC-FP6027748, www.bionets.eu

\section{REFERENCES}

[1] L. Pelusi, A. Passarella, and M. Conti, "Opportunistic networking: data forwarding in disconnected mobile ad hoc networks," IEEE Comm. Mag., vol. 44, no. 11, Nov. 2006.

[2] M. Nicolai, N. Behrens, and E. Yoneki, "Wireless rope: An experiment in social proximity sensing with bluetooth," in Proc. of PerCom, March 2006.

[3] X. Zhang, J. Kurose, B. N. Levine, D. Towsley, and H. Zhang, "Study of a Bus-Based Disruption Tolerant Network: Mobility Modeling and Impact on Routing," in Proc. of Mobicom, September 2007, pp. 195-206.

[4] BIONETS, "Biologically-inspired autonomic networks and services," http://www.create-net.eu, 2005.

[5] I. Carreras, D. Tacconi, and D. Miorandi, "Data-centric information dissemination in opportunistic environments," in In Proc. of MASS, Pisa, Italy, October 2007.

[6] I. Carreras, I. Chlamtac, F. D. Pellegrini, and D. Miorandi, "Bionets: Bio-inspired networking for pervasive communication environments," IEEE Trans. on Vehicular Technology, vol. 56, no. 1, pp. 218-229, Jan. 2007.

[7] Jsr-000118 mobile information device profile 2.0. [Online]. Available: http://jcp.org/aboutJava/communityprocess/final/jsr118/

[8] Jsr-000082 javatm apis for bluetooth. [Online]. Available: http://jcp.org/aboutJava/communityprocess/final/jsr082/ 